\title{
Beamstrahlung with fluctuating charge density
}

\section{Citation}

Jacob, M., and Tai Tsun Wu. 1989. "Beamstrahlung with Fluctuating Charge Density." Nuclear Physics B 314 (2): 334-46. https://doi.org/10.1016/0550-3213(89)90155-7.

\section{Permanent link}

http://nrs.harvard.edu/urn-3:HUL.InstRepos:41555801

\section{Terms of Use}

This article was downloaded from Harvard University's DASH repository, and is made available under the terms and conditions applicable to Other Posted Material, as set forth at http:// nrs.harvard.edu/urn-3:HUL.InstRepos:dash.current.terms-of-use\#LAA

\section{Share Your Story}

The Harvard community has made this article openly available.

Please share how this access benefits you. Submit a story.

Accessibility 


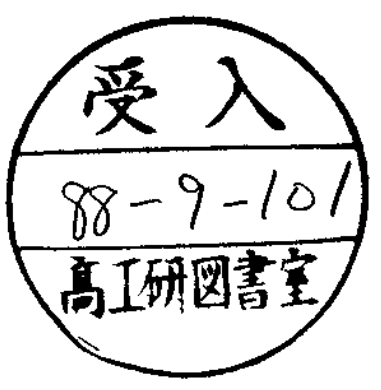

CERN-TH. $5133 / 88$

\section{BEAMSTRAHLUNG WITH FLUCTUATING CHARGE DENSITY}

M. Jacob

CERN - Geneva

and

Tai Tsun $\mathrm{Wu}^{*}$ )

CERN - Geneva

and

Gordon McKay Laboratory, Harvard University

\section{ABSTRACT}

The calculation of bremstrahlung during bunch-bunch collisions in the quantum regime, relevant to electron-positron colliders in the multi-TeV range, is extended to take into account fluctuations in bunch density.

*) Work supported in part by the United States Department of Energy under Grant DE-FG02-84 ER40158. 


\section{1. - INTRODUCTION}

In a recent series of papers [1-3], we studied the main properties of the radiation produced during the collision of two bunches of charged particles. The conditions considered were typical of those relevant for an electron-positron linear collider operating in the multi-TeV regime. Machines of that type are being actively considered as highly potential tools for high-energy physics [4,5]. It is well known that there is no point reaching very high energies if the luminosity is not high enough to compensate for the fall-off of the bench-mark annihilation cross-section with increasing energy. Reaching high luminosity implies very high bunch densities, and particles thus experience very strong accelerating fields as bunches cross each other. The intense bremsstrahlung which results is usually referred to as beamstrahlung [6].

The phenomenon is somehow special, since realistic machine parameters are such that this radiation is likely to occur in the deep quantum regime. In the classical treatment of synchroton radiation, the coherent radiation length $L_{c}=\rho_{c} / \gamma$, where $\rho_{c}$ is the radius of curvature of the electron path and $\gamma$ the usual Lorentz contraction factor $\mathrm{E} / \mathrm{m}$, is much larger than the radiative length $\mathrm{L}_{e}=\gamma / \mathrm{m}$, which can be altogether neglected. In the case now under consideration, it is the opposite which prevails, namely

$$
L_{e} \gg L_{c}
$$

This leads to a new radiation regime which has been the object of several recent investigations [4,7-9] together with ours [1-3].

All analytical approaches made so far refer to the low D approximation, where $D$ is the disruption parameter, the ratio between the bunch length $\mathrm{L}_{b}$ and the focal length associated with the focusing which a bunch imposes on impinging particles. In that approximation, the problem of bunch-bunch collisions can also be reduced to that of particle bunch collisions.

In our previous papers [1-3], we studied the beamstrahlung process in this deep quantum regime ( $\mathrm{L}_{e} \gg \mathrm{L}_{\mathrm{c}}$ ) through a Feymman graph approach which led us, to lowest order in $\alpha$, to a distorted wave Born approximation calculation of the radiation. We stressed the relevance of a new coherence length $\ell_{c}$, proper to that regime. It is defined as

$$
e_{c}^{3}=L_{c}^{2} L_{e}
$$

and condition (1) becomes 


$$
\left(\frac{l_{c}}{L_{c}}\right)^{2} \gg 1
$$

For reasons of simplicity, we considered a cylindrical uniform bunch, with charge density

$$
\rho=\left\{\begin{array}{cl}
\frac{N e}{\pi R^{2} L e} & |z|<\frac{L b}{2} \\
0 & |z|>\frac{L b}{2}
\end{array}\right.
$$

where $L_{b}$ and $R\left(L_{b} \gg R\right.$ ) are respectively the length and radius of the bunch which contains $N$ particles. In practice these values and the coherence properties of radiation are such that the granularity can be neglected.

In that case, we found two leading contributions to the fractional energy loss $\delta$, namely [3]

$$
\delta=\frac{\alpha}{\pi}\left(k_{1} \frac{L_{b}}{e_{c}}+k_{2} \ln \frac{L_{e}}{e_{c}}\right)
$$

where $K_{1}$ and $K_{2}$ are numexical factors of ordex one. This is relation (72) of Ref. [3], and this very simple formula is accurate when condition (1) holds [3,10]. The appearance of the second term and the full relevance of $l_{c}$, as opposed to $L_{c}$ ' are typical of the deep quantum regime. Typical values of $\delta$ are at the level of $20 \%[3,4]$.

The relevance of more realistic bunch shapes has been the object of recent investigations $[7,9,11]$. The purpose of this paper is to study this question with our distorted wave Born approximation approach. We first derive a general expression which is also suited to the eventual study of edge effects with realistic bunch shapes. Here we leave aside this important problem and apply our expression to the simpler problem of a bunch with fluctuating density. What we mean by that is a long bunch, of length $\mathrm{L}_{b}$, where the density is not constant but fluctuates within the bunch, with a typical length scale L', which may vary along the bunch, but with $L^{\prime} \ll L_{b}$.

\section{2. - BUNCH PROFILE AND RELATED EFEECTS}

We introduce a bunch profile function $\tilde{\rho}(z)$ through

$$
e(z)=\frac{N e}{\pi R^{2} L_{e}} \tilde{e}(z)
$$

where $L_{b}$ is now defined by 


$$
L_{b}=\int_{-\infty}^{+\infty} \tilde{e}(z) d z
$$

The fraction of the bunch charge encountered by an incident particle, up to $z$ (within the bunch) is

$$
\tau(z)=\frac{1}{L_{b}} \int_{-\infty}^{z} \tilde{e}(z) d z
$$

The leading effect can be easily derived heuristically. From (5), one may consider the first term as a sum of incoherent contributions originating from different successive lengths of order $l_{c}\left(l_{c} \ll L_{b}\right)$. Our expression for $l_{c}$ (relation (26) of Ref. [3]) is

$$
e_{c}=\left(\frac{R^{2} L_{l}^{2} k i}{(N \alpha)^{2}}\right)^{1 / 3}
$$

where $k_{i}$ is the incident electron energy. All variables are, in practice, defined In the bunch frame. With the "super" parameters [3,4], we have $L_{b} \approx 60 l_{c}$. Relation (9) applies to a constant density. If the density varies, $l_{c}$ will also vary. We have, from (9),

$$
e_{c}(z) \sim \tilde{e}^{-2 / 3}(z)
$$

As we have just done, we may first consider the approximation where the variation of $\tilde{\rho}$ is negligible over $\ell_{c}(z)$. This makes sense, since most of the radiation originates where $\tilde{p}(z)$ is the largest and accordingly $\ell_{c}(z)$ is the smallest. This approximation should thus apply to the most important part of the radiation.

We may then conclude that an important correction to the first term in (5), which results from a varying density, is merely to replace $K_{1}$ by a new factor $\bar{K}_{1}$, with

$$
\bar{k}_{1}=k_{1} \frac{\int \tilde{e}^{2 / 3}(z) d z}{\int \tilde{e}(z) d z}
$$

In many practical cases, namely density decreasing smoothly and uniformly from the centre, this corresponds to an increase in radiation. This is typical of the quantum regime. If we stretch the bunch Iength, the density decreases as $\mathrm{L}_{b}^{-1}$, but the local rate drops only as $\mathrm{L}_{\mathrm{b}}^{-2 / 3}$. The global rate thus increases as $\mathrm{L}_{\mathrm{b}}^{1 / 3}$. 
It is a straightforward matter to introduce such a local density at each step of our previous calculation $[2 ; 3\}$ and to reach (11) without this heuristic assumption [10]. The approximation which has to be made is that $\ell_{c}(z) \tilde{\rho}^{\prime}(z)<<\tilde{\rho}(z)$, thus neglecting the variation of the bunch density over a correlation length $l_{c}(z)$, which varies as $\tilde{\rho}(z)^{-2 / 3}$.

One may relax this approximation and introduce at each point the effect of $\tilde{\rho}^{\prime}$ and $\tilde{\rho} "$, though neglecting further derivatives. This is appropriate if there are smooth fluctuations of density along the bunch, with a typical length scale $L^{\prime}$ which, though larger than $\ell_{c}(z)$ since we remain deep inside the bunch where $\tilde{\rho}(z)$ is sizeable, may be much less than $\mathrm{L}_{b}$. It turns out that, as derived in Section 4, this results in a correction term to the local radiation yield proportional to

$$
R(z)=e_{c} \tilde{e}^{-2 / 3}(z)\left(\frac{4 \tilde{e}^{\prime 2}(z)-3 \tilde{e}(z) \tilde{e}^{\prime \prime}(z)}{\tilde{e}^{2}(z)}\right)
$$

The correlation length $\ell_{c}$ is here the nominal one (9), with $L_{b}$ defined by (7). All density variables are defined at a particular point $z$ and $R(z)$ refers to the amount of radiation originating from a length $\ell_{c}(z)$ around $z$. Since this radiation is practically incoherent from the rest of the radiation, it makes sense to consider it separately.

The last factor in (12) is proportional to $\left(L^{\prime}\right)^{-2}$. Since the leading contribution is of order $\left(1 / \ell_{c}\right) p^{2 / 3}(z)$, we thus obtatn a correction of relative order $\left(\ell_{c} / L^{\prime}\right)^{2}$, which may not be a very small quantity. We recall that with the "super" parameters, we have [2] $\mathrm{L}_{b} \gg l_{c}$. Hence $l_{c} \ll \mathrm{L}^{\prime} \ll \mathrm{L}_{b}$ is an interesting case.

This particular correction to the yield can then be integrated over practically all the bunch length, since it is built from non-interfering contributions over $\ell_{c}(z)$ long sections. However, it is not possible to use (12) to estimate the corrections due to the variation of density at the edge of a smooth-shaped bunch. While we would then have a very small $\left(10^{-3}\right)$ overall factor $\left(l_{c} / L_{b}\right)^{2}$, since $L_{b}$ may now set the scale for the variation of the density, the integral over the bunch diverges. It is not stralghtforward to isolate this particular contribution from others which originate coherently with it from regions of space at the edges of the bunch, where $\ell_{c}(z)$ becomes very large. Indeed, relation (12) is obtained within the assumption that $l_{c}(z) \ll L_{b}$. It is meaningful to use it inside the bunch, where separate sections of length $\ell_{c}(z)$ radiate incoherently. One may thus apply it to fluctuation of density inside the bunch.

The $\tilde{\rho}, \tilde{\rho}^{\prime}$ and $\tilde{\rho}^{\prime \prime}$ dependence of the correction term (12) agrees with the 
expression obtained by Bell and Bell [11]. The first-order correction (11) agrees with the result of Blankenbecler and Drell in their study of ribbon bunches [7]. Our approach, which follows our previous one $[2,3]$ is, however, different, and thus merits a special presentation. Some intermediate results are also potentially interesting for further studies.

\section{3. - BEAMSTRAHLUNG WITH RANGING DENSITY}

For reasons of simplicity, we shall work in the spinless case [2]. We indeed

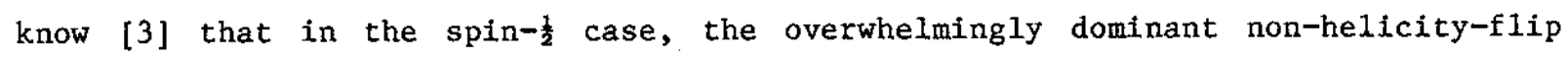
contribution is merely proportional, though with a different spectrum shape, to the contribution calculated in the spinless case [2]. The overall factor, related to the spin, is $\frac{1}{2}\left(1+(1-X)^{2}\right) /(1-X)$. This overall factor holds not only for the leading term but also for the effect of fluctuating charge density being studied here.

We introduce the two radiation matrix elements corresponding to photon polarization parallel and orthogonal to the classical bending plane, respectively. They are relations (33) of Ref. [2], but now written in terms of $\tau(z)$ (8), while the original ones [2] refer to a uniform bunch density. They read:

$$
\begin{aligned}
& m_{11}=\frac{2}{x}\left(x\left(k_{1 x}+h_{\gamma x}\right) \tau(z)-h_{\gamma x}\right) \\
& m_{\perp}=\frac{2}{x} k_{\gamma y}(x \tau(z)-1)
\end{aligned}
$$

We keep our previous notations [2,3]. The classical electron bending plane defines the $z, x$ plane. The electron has, after bunch-crossing, a momentum $k_{f}$, while the radiated photon, with fractional energy $X$, has a momentum $k_{\gamma}$, with only a relatively small component $k_{\gamma y}$ perpendicular to that plane. The radiation amplitude, at point $x, y, z$, has a phase $\phi(x, y, z)$, calculated in Ref. [2], and the full radiation amplitudes thus read

$$
M=\int_{-\infty}^{+\infty} d z \int_{r=0}^{R} d x d y e^{i \phi(x, y, z)}\left\{\begin{array}{l}
\frac{2}{x}\left(\left(k_{x}+h_{\gamma x}\right) x \tau(z)-h_{\gamma x}\right) \\
\frac{2}{x} k_{\gamma y}(x \tau(z)-1)
\end{array}\right.
$$

where the upper (lower) term refers to the polarization component parallel (perpendicular) to the classical bending plane.

The integral over the radial components $(x, y)$ is readily done using the stationary conditions derived in Ref. [2]. This gives 
$-6-$

$$
M=-i \pi \frac{R^{2}}{N \alpha} \int_{-\infty}^{+\infty} d z e^{i \phi(z)}\left\{\begin{array}{l}
\left.\left.\frac{2}{x}\left(h_{f_{x}}+h_{\gamma x}\right) x \tau / z\right)-h_{\gamma x}\right) \\
\frac{2}{x} h_{\gamma y}(x \tau(z)-1)
\end{array}\right.
$$

As discussed in Ref. [2], there is no proper stationary point in $z$. The phase is indeed such that [2]

$$
\frac{\partial \phi}{\partial z}=\frac{1}{2 x(1-x) h_{i}}\left\{\left(\left(h_{f x}+h_{\gamma x}\right) x \tau(z)-h_{\gamma x}\right)^{2}+h_{\gamma y}^{2}\left(x(\tau(z)-1)^{2}+x_{m^{2}}^{2}\right\}\right.
$$

which, using (13), can also be written as

$$
\frac{\partial \phi}{\partial z}=\frac{x}{8(1-x) k_{i}}\left\{\left|m_{11}\right|^{2}+\left|m_{\perp}\right|^{2}+4 m^{2}\right\}
$$

We have kept here the relatively small $\mathrm{m}^{2}$ term which was systematically neglected in Ref. [2].

The phase of the radiation amplitude can then be written, up to an irrelevant constant term, as:

$$
\begin{aligned}
\phi(z)= & \frac{1}{2 x(1-x) k_{i}}\left\{\left(\left(k_{f x}+h_{\gamma x}\right)^{2}+h_{\gamma y}^{2}\right) x^{2} T(z) L_{b}\right. \\
& -2\left(h_{\gamma x}\left(h_{f x}+h_{\gamma x}\right)+h_{\gamma y}^{2}\right) \times U(z) L_{b} \\
& \left.+\left(h_{\gamma x}^{2}+h_{\gamma y}^{2}+x^{2} m^{2}\right) z\right\}
\end{aligned}
$$

where

$$
\begin{gathered}
T(z)=\frac{1}{L_{b}} \int_{-\infty}^{z} \tau^{2}\left(z^{\prime}\right) d z^{\prime} \\
U(z)=\frac{1}{L_{b}} \int_{-\infty}^{z} \tau\left(z^{\prime}\right) d z^{\prime}=\frac{1}{L_{l}^{2}} \int_{-\infty}^{z} \tilde{e}\left(z^{\prime}\right)\left(z-z^{\prime}\right) d z^{\prime}
\end{gathered}
$$


$-7-$

The radiation rate is obtained taking the modulus squared of (15) and integrating over phase space, namely

$$
I=\frac{4 \pi \alpha}{8(2 \pi)^{5} k_{i}} \int|M|^{2} \delta\left(k_{i}-k_{f}-k_{\gamma}\right) \frac{d^{3} \hat{h}_{f} d^{3} h_{\gamma}}{k_{f} k_{\gamma}}
$$

where $|M|^{2}$ stands for $\left|M_{\|}\right|^{2}+\left|M_{\perp}\right|^{2}$.

Using (15) and (18), and doing all the trivial integrals, I can be rewritten as

$$
\begin{aligned}
& I=\frac{1}{8(2 \pi)^{2} k_{i}^{2}}\left(\frac{R^{2}}{2 N \alpha}\right)^{2} \int_{0}^{1}\left(\frac{2}{x}\right)^{2} \frac{d x}{x(1-x)} \\
& \int_{-\infty}^{+\infty} d z \int_{-\infty}^{+\infty} d z^{\prime} e^{i \frac{x m^{2}}{2(1-x) k_{i}}\left(z-z^{\prime}\right)} F\left(z, z^{\prime}\right)
\end{aligned}
$$

where

$$
\begin{aligned}
& \left.F\left(z, z^{\prime}\right)=\int_{f_{x} d k_{f_{x}} d k_{\gamma_{x}} d k_{\gamma y}} e^{i\left(\tilde{\phi}(z)-\tilde{\phi}\left(z^{\prime}\right)\right.}\right) \\
& \left.\left\{\left(\left(h_{f_{x}}+h_{\gamma x}\right) \times \tau / z\right)-h_{\gamma x}\right)\left(\left(h_{f x}+h_{\gamma x}\right) \times \tau / z^{\prime}\right)-h_{\gamma x}\right) \\
& \left.+h_{\gamma y}^{2}(x \tau(z)-1)\left(x \tau\left(z^{\prime}\right)-1\right)\right\}
\end{aligned}
$$

Here $\tilde{\phi}(z)$ is the phase as previously obtained (18), but with the $\mathrm{m}^{2}$ contribution subtracted and factored out separately in (21).

Relations (21) and (22) constitute the starting point of our present calculaLion. It is easy to check [3] that (22) also applies to the Dirac case, when summing and averaging over polarization and neglecting helicity flip, as fully justified in the deep quantum regime. One then simply includes the extra factor $\frac{1}{2}\left(\left(1+(1-x)^{2}\right) /(1-X)\right)$ in the integrand in (21).

We here depart from our previous approach [2,3], where we used the stationary phase conditions to calculate the $z$ integrals in terms of Airy functions and took the modulus squared of the amplitude before performing the phase space integrals. Now we first perform the phase space integrals, leaving to the end the integrals over both $z$ and $z^{\prime}$. 
$-8-$

We use the relation between the derivative of $\phi$ and the radiation matrix elements (17) to simplify the calculation of the phase space integral in (22). This is done as follows. Write $\tilde{\phi}(z)-\tilde{\phi}\left(z^{\prime}\right)$ as (18)

$$
\begin{aligned}
\tilde{\phi}\left(z, z^{\prime}\right)= & \left(\left(h_{f x}+h_{\gamma x}\right)^{2}+h_{\gamma y}^{2}\right) A+\left(h_{\gamma x}\left(h_{f x}+h_{\gamma x}\right)+h_{\gamma y}^{2}\right) B \\
& +\left(h_{\gamma x}^{2}+h_{\gamma y}^{2}\right) C
\end{aligned}
$$

where

$$
\begin{aligned}
& A=\frac{1}{2 x(1-x) h_{i}} x^{2} L_{G}\left(T(z)-T\left(z^{\prime}\right)\right) \\
& B=\frac{1}{2 \times(1-x) h_{i}}\left(-2 x \operatorname{Lg}\left(U(z)-U\left(z^{\prime}\right)\right)\right) \\
& C=\frac{1}{2 \times(1-x) h_{i}}\left(z-z^{\prime}\right)
\end{aligned}
$$

and introduce a quantity

$$
\begin{gathered}
F_{0}\left(z, z^{\prime}\right)=\int h_{f_{x}} d h_{f_{x}} d h_{\gamma_{x}} d h_{\gamma_{y}} e^{i \tilde{\phi}\left(z^{\prime} z^{\prime}\right)} \\
=\frac{1}{2 \pi} \int d h_{f_{x}} d h_{f_{y}} d h_{\gamma_{x}} d h_{\gamma_{y}} e^{i \tilde{\phi}\left(z^{\prime} z^{\prime}\right)}
\end{gathered}
$$

thus restoring explicitly the symmetry through invariance under rotation of the classical bending plane.

It then follows that

$$
F\left(z, z^{\prime}\right)=-i\left\{x^{2} \tau(z) \tau\left(z^{\prime}\right) \frac{\partial}{\partial A}-x\left(\tau(z)+\tau\left(z^{\prime}\right)\right) \frac{\partial}{\partial B}+\frac{\partial}{\partial C}\right\} F_{0}\left(z, z^{\prime}\right)
$$

and

$$
\begin{gathered}
\tilde{\phi}\left(z, z^{\prime}\right)=\left(\Delta_{T x}^{2}+\Delta_{T y}^{2}\right) A-\left(h_{\gamma x} \Delta_{T x}+h_{\gamma y} \Delta_{T y}\right) B \\
+\left(h_{\gamma x}^{2}+h_{\gamma y}^{2}\right) C
\end{gathered}
$$

where, following Ref. [2], we have introduced the components of the global transfired momentum to the bunch 
- 9 -

$$
\Delta_{T}=-\left(h_{f}+h_{\gamma}\right)
$$

The phase space integrals are limited to the region [2]

$$
\Delta_{T}^{2}<\left(\frac{2 N \alpha}{R}\right)^{2}
$$

Using new variables

$$
\begin{aligned}
& \bar{h}_{\gamma x}=h_{\gamma x}-\frac{B}{2 C} \Delta_{T x} \\
& \bar{h}_{\gamma y}=h_{\gamma y}-\frac{B}{2 C} \Delta_{T y}
\end{aligned}
$$

one can then perform all phase space integrals in (25) with $\tilde{\phi}$ given by (26). This gives

$$
F_{0}\left(z, z^{\prime}\right)=\frac{2 \pi}{4 A C-B^{2}}\left(1-e^{i\left(\frac{2 N \alpha}{R}\right)^{2} \frac{4 A C-B^{2}}{4 C}}\right)
$$

No approximation has been made at this stage. The integrals over $\overline{\mathrm{k}}_{\gamma_{\mathrm{x}}}$ and $\overline{\mathrm{k}}_{\gamma_{\mathrm{y}}}$ are extended to infinity, but this is perfectly legitimate in view of the limitations imposed on these momentum components.

We now introduce a simplifying trick in the calculation of $F\left(z, z^{\prime}\right)(26)$. As is easily checked (24)

$$
\begin{aligned}
& \frac{\partial}{\partial z} \equiv \frac{1}{2 x(1-x) h_{i}}\left(x^{2} \tau^{2}(z) \frac{\partial}{\partial A}-2 x \tau(z) \frac{\partial}{\partial B}+\frac{\partial}{\partial C}\right) \\
& \frac{\partial}{\partial z^{\prime}} \equiv \frac{1}{2 x(1-x) h_{i}}\left(x^{2} \tau^{2}\left(z^{\prime}\right) \frac{\partial}{\partial A}-2 x \tau\left(z^{\prime}\right) \frac{\partial}{\partial B}+\frac{\partial}{\partial C}\right)
\end{aligned}
$$

when operating on $\mathrm{F}_{\mathrm{o}}\left(z, z^{\prime}\right)$. It therefore follows that

$$
\begin{gathered}
F\left(z^{\prime} z^{\prime}\right)=-2 \pi i\left(x^{2} \tau(z) \tau\left(z^{\prime}\right) \frac{\partial}{\partial A}+\frac{1}{2}\left(\frac{\partial}{\partial z}+\frac{\partial}{\partial z^{\prime}}\right) 2 x(1-x) \xi_{i}\right. \\
\left.-\frac{x^{2}}{2}\left(\tau^{2}(z)+\tau^{2}\left(z^{\prime}\right)\right) \frac{\partial}{\partial A}\right) F_{0}\left(z, z^{\prime}\right)
\end{gathered}
$$


Through integration by parts of (21), the second term will give a term proportional to $\mathrm{m}^{2}$ and can therefore be neglected in the following, when we focus on radiation from inside the bunch. What remains is only a differentiation with respect to $A$ and we can write $(22)$ as

$F\left(z, z^{\prime}\right)=i \pi x^{2}\left(\left(\tau(z)-\tau\left(z^{\prime}\right)\right)^{2} \frac{\partial}{\partial A} \frac{1}{4 A C-B^{2}}\left(1-e^{i\left(\frac{2 N \alpha}{R}\right)^{2} \frac{4 A C-B^{2}}{4 C}}\right)\right.$

with $A, B$ and $C$ given by (24).

We have therefore an expression for the radiation rate $I$ (21) as a double integral over the bunch axis. Neglecting the $\mathrm{m}^{2}$ term, it reads:

$$
\begin{gathered}
I=\frac{4 \pi \alpha}{8(2 \pi)^{2} k_{i}^{2}}\left(\frac{R^{2}}{2 N \alpha}\right)^{2} \int_{0}^{1}\left(\frac{2}{x}\right)^{2} \frac{d x}{x(1-x)} \\
\int_{-\infty}^{+\infty} d z \int_{-\infty}^{+\infty} d z^{\prime} F\left(z, z^{\prime}\right)
\end{gathered}
$$

with $F\left(z, z^{\prime}\right)$ given by (34). This can be used to calculate all the different components of the radiation rate, with arbitrary bunch profiles.

The $\mathrm{m}^{2}$ terms should also be introduced for a full study of radiation, since they contribute in a relatively important way to the integral over the region outside the bunch. This was considered separately in Ref. [1] for the case of a sharp boundary, when the second term in (5) was derived.

In this paper we only consider the effect of density fluctuations deep inside the bunch. We thus start with (34) and (35) and use our previous result [2] that the phase conditions impose a strong coherence condition, with radiation originating from a length $\ell_{c}(z)$ around a point $z$ defined by the final momenta. Instead of integrating over $z$ and $z^{\prime}$ varying along the whole axis, we integrate on $z=\left(z+z^{\prime}\right) / 2$ and on $\mathscr{\zeta}=z-z^{\prime}$, eventually expanding in $\mathscr{\zeta}$. The stationary phase conditions are such that the radiation damps itself out outside a range of $C_{f}$ which is much smaller than $\mathrm{L}_{b}$. This of course applies only as long as $\tilde{\rho}(z)$ keeps a relatively large value, so that $\ell_{c}(z)$ remains small as compared to $L_{b}$. 


\section{4. - THE INFLUENCE OF DENSITY FLUCTUATIONS}

We can write (34) as

$$
F\left(z, z^{\prime}\right)=i \pi x^{2}\left(\left(\tau(z)-\tau / z^{\prime}\right)\right)^{2} \frac{1}{4 C} \int_{0}^{\left(\frac{2 N \alpha}{R}\right)^{2}} y d y e^{i y \frac{4 A C-B^{2}}{4 C}}
$$

so that the only terms needed are $\left(4 A C-B^{2}\right) / 4 C$ and $C$.

For $\mathrm{C}$, we have

$$
C=\frac{1}{2 x(1-x) k_{i}} \mathscr{G}
$$

but for $\left(4 \mathrm{AC}-\mathrm{B}^{2}\right) / 4 \mathrm{C}$, we have to expand in powers of $\mathscr{G}$. We shall include explicitly the effect of $\tilde{\rho}^{\prime}$ and $\tilde{\rho}^{\prime \prime}$ terms, which corresponds eventually to our including terms of order $\tau^{\prime 2}, \tau^{\prime} \tau^{\prime \prime \prime}$ and $\tau^{\prime 2}$. We thus deal with fluctuations which are relatively smooth over $l_{c}$ but quite sizeable over $L_{b}$. One finds (to order $\varphi^{5}$ )

$\frac{4 A C-B^{2}}{4 C}=\frac{x}{2(1-x) h_{i}} \varphi^{3}\left(\frac{\tau^{\prime 2}}{12}+\frac{1}{720}\left(3 \tau^{\prime} \tau^{\prime \prime \prime}+\tau^{\prime \prime 2}\right) \varphi^{2}\right)$

All density functions are defined at point $z=\left(z+z^{\prime}\right) / 2$. One can then rewrite (34) as

$$
\begin{gathered}
F\left(z, \mathscr{G}_{1}\right)=i \frac{\pi}{2} x^{3}(1-x) h_{i} \zeta\left(\tau^{\prime 2}+\frac{1}{12} \tau^{\prime} \tau^{\prime \prime \prime} \varphi^{2}\right) \\
\int_{0}^{\left(\frac{2 N \alpha}{R}\right)^{2}} \eta d \eta e^{i \eta U}
\end{gathered}
$$

with

$$
U=\frac{x}{2(1-x) h_{i}} \xi^{3}\left(\frac{\tau^{\prime 2}}{12}+\frac{1}{720}\left(3 \tau^{\prime} \tau^{\prime \prime \prime}+\tau^{\prime \prime 2}\right) \xi^{2}\right)
$$

where we have used

$$
\left(\tau(z)-\tau\left(z^{\prime}\right)\right)^{2}=\mathscr{G}^{2}\left(\tau^{\prime 2}+\frac{1}{12} \tau^{\prime} \tau^{\prime \prime \prime} \varphi^{2}\right)
$$

We can then perform the integral over $\zeta$ as implied by (35). We simplify it through the change of variable 


$$
\xi=\xi+\alpha \xi^{3}, \alpha=\frac{3 \tau^{\prime} \tau^{\prime \prime \prime}+\tau^{\prime 2}}{180 \tau^{\prime 2}}
$$

so that, up to consistently-kept $\xi^{3}$ terms, we have

$$
\begin{aligned}
& \int_{-\infty}^{+\infty} d z \int_{-\infty}^{+\infty} d z^{\prime} F\left(z^{\prime} z^{\prime}\right)=\int d z \frac{i \pi}{2} x^{3}(1-x) k_{i} \int_{0}^{\left(\frac{2 N \alpha}{R}\right)^{2}} y d y \\
& \int \xi d \xi \tau^{\prime 2}\left(1-\xi^{2} \frac{4 \tau^{\prime \prime 2}-3 \tau^{\prime} \tau^{\prime \prime \prime}}{180 \tau^{\prime 2}}\right) e^{i \eta \frac{x \xi^{3}}{24(1-x) k_{i}} \tau^{\prime 2}}
\end{aligned}
$$

As previously stressed, this expression is reliable deep inside the bunch only. The overall effect results from an integral extended over most of the bunch length $\mathrm{L}_{\mathrm{b}}$. It applies to fluctuation densities within the bunch.

For the maximum value of $\eta=2 \mathrm{~N} \alpha / \mathrm{R}$, the argument in the exponential is

$$
\left(\frac{2 N \alpha}{R}\right)^{2} \frac{x}{2(1-x) h_{i}} \frac{1}{12 L_{l}^{2}} \tilde{e}^{2}(\xi) \xi^{3}
$$

where one recognizes a factor $\xi^{3} / l_{c}^{3}(z)(9)$, (10). The integral over $\xi$ thus translates the coherence conditions previously used when calculating the radiation amplitude [2].

We now proceed with the integral, introducing

$$
\beta=\frac{x}{2(1-x) h_{i}} \frac{\tau^{12}}{12} \eta
$$

and

$$
y=-i \beta \xi^{3}
$$

The integrals over $\xi$ are of two kinds

$$
\begin{aligned}
& \int e^{i \beta \xi^{3}} \xi d \xi=\frac{1}{\sqrt{3}} \beta^{-2 / 3} \int_{0}^{\infty} y^{-1 / 3} e^{-y} d y=\frac{1}{\sqrt{3}} \beta^{-2 / 3} \Gamma(2 / 3) \\
& \int e^{i \beta \xi^{3}} \xi^{3} d \xi=\frac{1}{\sqrt{3}} \beta^{-4 / 3} \int_{0}^{\infty} y^{1 / 3} e^{-y} d y=\frac{1}{\sqrt{3}} \beta^{-4 / 3} \Gamma(4 / 3)
\end{aligned}
$$

The integral over $\eta$ can then be performed easily. We have respectively 


$$
\begin{aligned}
& \int_{0}^{\left(\frac{2 N \alpha}{R}\right)^{2}} \eta^{1 / 3} d y=\frac{3}{4}\left(\frac{2 N \alpha}{R}\right)^{8 / 3} \\
& \int_{0}^{\left(\frac{2 N \alpha}{R}\right)^{2} \eta^{-1 / 3} d y=\frac{3}{2}\left(\frac{2 N \alpha}{R}\right)^{4 / 3}}
\end{aligned}
$$

We can now use the expression obtained for (43) in (35) and calculate the radiation rate from deep inside the bunch. It is a leading part, corresponding to an integral over the bunch length, and separated from the rest by the coherence conditions.

We write the spectrum normalized to unit area $J(x)$. The integral over this spectrum, weighted by $\mathrm{x}$, then gives the fractional energy loss $\delta[2,3]$.

For the first term, involving the density only, we have:

$$
J_{l}(x)=\frac{\alpha}{\pi} \frac{6^{2 / 3} \sqrt{3}}{4} r(2 / 3)\left(\frac{1-x}{x}\right)^{2 / 3} \frac{1}{e_{c}} \int \tilde{\rho}^{2 / 3}(z) d z
$$

We thus reproduce our previous result with $K_{1}=1.94$ [2], but with the correction (11). The radiation has a spectrum $((1-X) / X)^{2 / 3}$. The rate involves, as expected, a factor $\alpha / \pi$, a numerical factor of order 1 , and a term of order $L_{b} / l_{c}$.

For the second term, involving the factor $\mathrm{R}$ (12), which is globally of order $\left(\ell_{c} / L^{\prime}\right)^{2} L_{b} / \ell_{c}$, we have

$$
\begin{aligned}
J_{2}(x)= & \frac{x}{\pi} \frac{\sigma^{4 / 3} \sqrt{3}}{2} r(4 / 3)\left(\frac{1-x}{x}\right)^{4 / 3} e_{c} \\
& \int \tilde{e}^{-2 / 3}(z) \frac{3 \tilde{e}(z) \tilde{e}^{\prime \prime}(z)-4 \tilde{e}^{\tilde{\prime}(z)}}{180 \tilde{e}^{2}(z)} d z
\end{aligned}
$$

The spectrum is softer, having an extra factor $((1-X) / X)^{2 / 3}$. One obtains an integral over $R(Z)$ (12). It has to be cut off to remain within the region where $\ell_{c}(z) \ll L_{b}$, but $i t$ is globally of order $L_{b}$. When the first contribution is of order $(\alpha / \pi) \mathrm{L}_{b} / \ell_{c}$, the second one is of order $(\alpha / \pi)(1 / 180)\left(\ell_{c} / L^{\prime}\right)^{2} L_{b} / \ell_{c}$. It can therefore be deemed a small correction term.

These results also apply to the Dirac case when neglecting helicity flip [3] The extra factor $\frac{1}{2}\left(\left(1+(1-X)^{2}\right) /(1-X)\right)$ has to be included in the spectrum. 


\section{ACKNOWLEDGEMENTS}

We are grateful to Joachim Bartels and John Bell for helpful discussions. One of us (T.T.W.) wishes to thank the CERN Theory Division for its kind hospitality.

\section{REFERENCES}

[1] M. Jacob and T.T. Wu, Phys. Lett. B197 (1987) 253.

[2] M. Jacob and T.T. Wu, Nuc1. Phys. B303 (1988) 373.

[3] M. Jacob and T.T. Wu, Nucl. Phys. B303 (1988) 389.

[4] R. Blankenbecler and S. Dre11, Phys. Rev. B36 (1987) 277.

[5] B. Richter, Nucl. Inst. and Methods 136 (1976) 47;

B. Richter, Proceedings of the 1984 ICFA Seminar on Future Perspectives in High-Energy Physics;

U. Amaldi, Phys. Lett. 61B (1976) 313;

$\mathrm{U}$. Amaldi, "Introduction to the Next Generation of Linear Colliders", CERN-EP 87-169 (1987).

[6] T. Himel and J. Siegrist, AlA Conference Proceedings 130 (1985); R.J. Noble, SLAC-PUB-3871 (1986).

[7] R. Blankenbecler and S. Dre11, SLAC-PUB-4883 (1987).

[8] M. Bell and J.S. Bell, CERN PS 87-53 (1987);

M. Bell and J.S. Bell, CERN-TH.4936/87 (1987).

[9] P. Chen, SLAC PUB-4391 (1987);

P. Chen and K. Yokoga, SLAC-PUB-4597 (1988).

[10] For a general summaxy of our previous work, see: M. Jacob, CERN-TH.5041/88 (1988), Proceedings of the 1988 Kazimierz Conference (World Scientific, to be published).

[11] M. Bel1 and J.S. Bell, CERN-TH.5056/88 (1988). 